\title{
A REVIEW OF THEORY
}

\section{J. SEATON}

Dept. of Physics, University College London, England

In the present review I will discuss certain rather general aspects of the theory, and will leave it to other speakers to describe the results of detailed calculations. Further references to recent work are given in the Report of IAU Commission 14.

\section{Atomic Wave Functions and Energy Levels}

In order to calculate data of importance for ultra-violet and X-ray astronomy wavelengths, transition probabilities, collision cross-sections, ionization and recombination rates, and line-broadening parameters - it is necessary to begin with the calculation of atomic wave functions. The interest is mainly in highly ionized systems.

The non-relativistic Schroedinger equation for an atom with $N$ electrons and nuclear charge $Z$ is

$$
\left\{-\frac{1}{2} \sum_{i=1}^{N} \nabla_{i}^{2}+V_{n e}+V_{e e}\right\} \Psi=E \Psi
$$

where $E$ is the energy in atomic units $(27.2 \mathrm{eV})$,

$$
V_{n e}=-\sum_{i=1}^{N} \begin{aligned}
& Z \\
& r_{i}
\end{aligned}
$$

is the potential energy of the electrons in the field of the nucleus, and

$$
V_{e e}=\sum_{j=i+1}^{N} \sum_{i=1}^{N-1} \frac{1}{r_{i j}}
$$

is the potential energy of the electron-electron interactions. The summation in $V_{n e}$ contains $N$ terms and the double summation in $V_{e e}$ contains $\frac{1}{2} N(N-1)$ terms. In order of magnitude the importance of $V_{e e}$ relative to $V_{n e}$ is therefore

$$
\left|\frac{V_{e e}}{V_{n e}}\right| \simeq \frac{(N-1)}{2 Z} .
$$

For highly ionized systems, $Z \gg N, V_{e e}$ may be treated as a small perturbation. The zero-order equation, neglecting $V_{e e}$ altogether, is

$$
\left\{-\frac{1}{2} \sum \nabla_{i}^{2}+V_{n e}\right\} \Psi_{0}=E_{0} \Psi_{0}
$$


This equation has solutions

$$
\Psi_{0}=\prod_{i=1}^{N} \psi_{\alpha_{i}}\left(\mathbf{x}_{i}\right)
$$

where $\mathbf{x}_{i}$ is the space and spin coordinate of electron $i,\left(\mathbf{r}_{i}, \sigma_{i}\right)$, and $\alpha_{i}$ stands for the set of one-electron quantum numbers, $\left(n_{i} l_{i} m_{s i} m_{l i}\right)$. The $\psi_{\alpha_{i}}$ are one-electron hydrogenic functions, satisfying the equation

$$
\left\{-\frac{1}{2} \nabla^{2}-\frac{Z}{r}\right\} \psi_{\alpha_{i}}=\varepsilon_{i} \psi_{\alpha_{i}}
$$

where

$$
\varepsilon_{i}=-\frac{1}{2} \frac{Z^{2}}{n_{i}^{2}} .
$$

The total zero-order energy in (5) is

$$
E=\sum_{i} \varepsilon_{i}=-\frac{Z^{2}}{2} \sum_{i} \frac{1}{n_{i}^{2}} .
$$

The contribution of $V_{e e}$ to the energy can be calculated using perturbation theory:

$$
E_{1}=E_{0}+\left(\Psi_{0}\left|V_{e e}\right| \Psi_{0}\right) .
$$

The complication immediately arises that the zero-order problem can be highly degenerate, that is to say there can be many quantum states $\Psi_{0}$ with the same energy $E_{0}$. Following Layzer (1959), two states are said to belong to the same complex if they have the same set of principal quantum numbers $n_{i}$ and the same parity. In applying (10) it is necessary to consider all states $\Psi_{0}$ which belong to the same complex, and which therefore have the same zero-order energy, and to diagonalize the matrix of $V_{e e}$ with respect to these states. The states $\Psi_{0}$ must, of course, be properly anti-symmetrized, so as to satisfy the Pauli exclusion principle.

Extensive calculations have been made by Godfredsen (1966) using hydrogenic zeroorder functions. An improvement can be obtained on making an approximate allowance for $V_{e e}$ in the zero-order problem. Eissner and Nussbaumer (1969) use a centralfield potential

$$
V_{\mathrm{cf}}=\sum_{i} v_{\mathrm{cf}}\left(r_{i}\right)
$$

and solve the zero-order problem

$$
\left\{-\frac{1}{2} \sum_{i} \nabla_{i}^{2}+V_{\mathrm{cf}}\right\} \Psi_{0}=E_{0} \Psi_{0} .
$$

The first-order energy is calculated as

$$
E_{1}=\left(\Psi_{0}|H| \Psi_{0}\right)
$$

where $H$ is the Hamiltonian operator in Equation (1). In this approach one includes 
all states $\Psi_{0}$ which have energies $E_{0}$ close together, and diagonalizes the matrix of $H$ with respect to these states.

Let us consider an iso-electronic sequence ( $N$ fixed, $Z$ variable). As $Z$ increases one will obtain an improved agreement between the calculated energies $E_{1}$ and the exact energies $E$ for the non-relativistic Schroedinger problem (1). This is a consequence of (4). However, on comparing calculated energies with observed energies it is found that the agreement is generally good for systems a few times ionized, but less good for highly ionized systems. This is clearly due to neglect of relativistic effects in the calculations.

Recent work has led to some major improvements in the calculation of relativistic corrections. One approach has been to include relativistic terms in the calculation of the zero-order wave functions; thus, for example, the one-electron Schroedinger equation (7) could be replaced by the one-electron Dirac equation. This approach should give good results for systems which are very highly ionized (it is exact for ions containing only one electron). Another approach is to obtain fairly accurate solutions of the $\mathrm{N}$-electron non-relativistic problem, and to allow for relativistic corrections to the energy using perturbation theory. Other speakers will describe results obtained using these methods.

\section{Radiative Transition Probabilities}

Extensive tabulations of transition probabilities are available (Wiese et al., 1966) and a Data Center on Transition Probabilities is operated by the National Bureau of Standards, Washington.

Calculations are fairly simple for transitions which may be considered to involve a single outer electron. One may use the Coulomb approximation of Bates and Damgaard (1949) or more refined calculations in which a central potential is adjusted in such a way as to give agreement between calculated and observed one-electron binding energies (see, for example, Stewart and Rotenberg, 1965).

For more complicated cases the problem of calculating transition probabilities must be considered as an extension of the problem of calculating wave functions; once the wave functions are known the transition probabilities can be computed without much effort. The general theory is reviewed by Layzer and Garstang (1968).

For many transition probability calculations it is necessary to include spin and other relativistic effects. This is essential, for example, if one wishes to obtain correct relative intensities for the components of multiplets, or if one is concerned with forbidden transitions.

\section{Electron-Ion Collision Cross-Sections}

Classical theory (see Burgess and Percival, 1968) may be used to estimate crosssections for collisional ionization and for transitions between highly excited states of hydrogenic ions. For the calculation of cross-sections for optically allowed 
transitions in positive ions extensive use has been made of results based on semiclassical impact parameter theories (the ion is treated quantum mechanically but the colliding electron is assumed to follow a classical path). These theories give expressions such that the cross-section is proportional to the optical oscillator strength. The formula involving a factor $\bar{g}$ should give results correct to a factor of 2 or so for transitions having reasonably large oscillator strengths (Van Regemorter, 1962). An improved, but more elaborate, semi-classical theory is discussed by Burgess et al. (1970).

All classical and semi-classical cross-section approximations have severe limitations. Astronomers often ask for some simple approximate formula for the calculation of collision cross-sections. I think the short answer is that there is no simple formula which is adequate for the interpretation of the large amount of good observational data which is now available. For collision cross sections, as for transition probabilities, it is necessary to make much more accurate quantum mechanical calculations. Fortunately, such calculations are easier for positive ions than for neutral atoms.

The wave function $\Psi$ for the electron-atom system can be expanded in the form where:

$$
\Psi=\mathscr{A} \sum \Psi_{A} \varphi_{A}
$$

$\mathscr{A}$ is an anti-symmetrization operator (anti-symmetrization implies allowance for electron exchange, which can give transitions involving a change in ion spin)

$\sum$ is a summation over states of the target system

$\Psi_{A}$ is a wave function for the target system

$\varphi_{A}$ is an orbital function for the colliding electron.

Using a variational theory for the determination of the 'best' cross-sections one obtains a set of coupled integro-differential equations for the functions $\varphi_{A}-$ this is the so-called 'close-coupling' approximation. For many near-threshold transitions in neutral atoms no approximation significantly simpler than the close coupling method can be expected to give accurate results, but for highly ionized systems some simplification can be made. It is essential that the functions $\varphi_{A}$ should be calculated using a potential with correct asymptotic form. If these functions are calculated neglecting coupling between the target states, we have equations of the form

where

$$
\left\{-\frac{1}{2} \nabla^{2}+v(r)\right\} \varphi_{A}=\frac{1}{2} k_{A}^{2} \varphi_{A}
$$

$$
v(r) \underset{r \rightarrow \infty}{\sim}-z / r
$$

and where $z=(Z-N)$ is the charge on the ion. In (15), $\frac{1}{2} k_{A}^{2}$ is the kinetic energy of the colliding electron when the target system is in state A. In the Coulomb-Born approximation one uses the potential $v=-z / r$ and in the distorted wave approximation one uses a central-field potential which has the behaviour (16) for $r$ large and which behaves like $-Z / r$ for $r$ small. Some refinements in the distorted wave method are discussed by Saraph et al. (1969). 
Using solutions of (15) one constructs wave functions $\Psi_{i}, \Psi_{f}$ for the electron-ion system in the initial state and the final state. The collision cross-section $Q(i \rightarrow f)$ is proportional to

$$
\left|\left(\Psi_{f}|H-E| \Psi_{i}\right)\right|^{2}
$$

where $H$ is the total Hamiltonian and $E$ the total energy. Results are often expressed in terms of collision strengths $\Omega$ :

$$
Q(i \rightarrow f)=\frac{\Omega(i, f)}{k_{i}^{2} \omega_{i}} \pi a_{0}^{2}
$$

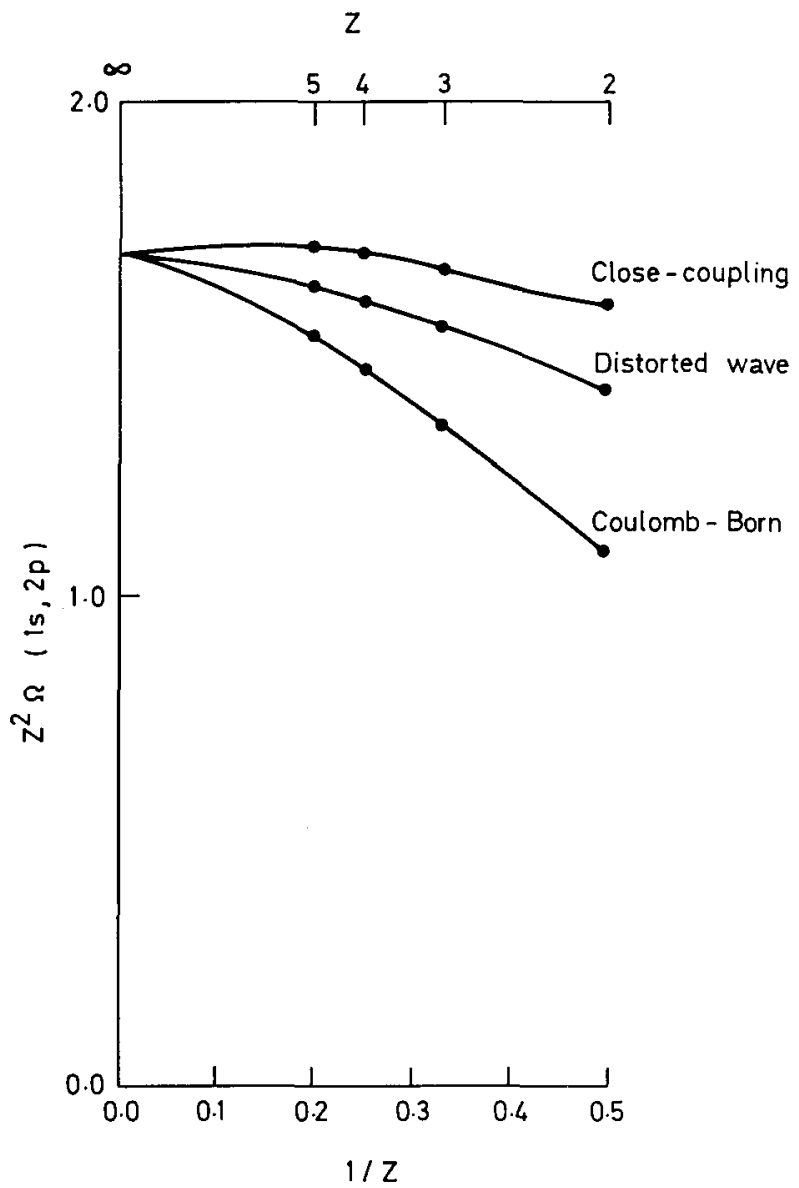

Fig. 1. Collision strengths for $1 s-2 p$ transitions in hydrogenic ions calculated by Belling (1970) in the 'close-coupling' approximation and in distorted wave and

Coulomb-Born approximations, including exchange. 
where:

$Q$ is the cross-section

$\frac{1}{2} k_{i}^{2}$ is the initial kinetic energy of the colliding electron, in atomic units

$\omega_{i}$ is the statistical weight of the initial level of the target system

$a_{0}$ is the Bohr radius.

The collision strength has the following properties: (i) $\Omega(i, f)=\Omega(f, i)$; (ii) for positive ions $\Omega$ remains finite at the excitation threshold; (iii) $Z^{2} \Omega$ remains finite in the limit of $Z \rightarrow \infty$. In an iso-electronic sequence one may plot $Z^{2} \Omega$, for a fixed value of $\left(k_{i} / Z\right)^{2}$, as a function of $1 / Z$. Figure 1 gives some recent results obtained by Belling (1970); for $N=1, Z^{2} \Omega(1 s, 2 p)$ is plotted as a function of $1 / Z$ for $\left(k_{1} / Z\right)^{2}=1.0$. The distorted wave method is seen to give good agreement with the close-coupling calculations for all positive ions in the sequence. The Coulomb-Born method is not quite so good but still gives reasonable results. In other sequences it is sometimes found that the distorted wave method may give results in error by a factor of 2 or so for singly-ionized positive ions, but better accuracy for more highly ionized systems.

To summarize, I would say that it is now possible to obtain reasonably accurate cross-sections for excitation of positive ions, although a fair amount of computation may be involved. It is possible to make some allowance for relativistic effects in the ion wave functions used in atomic collision calculations, but little work has so far been done on the possible importance of relativistic effects in the collision process itself.

\section{References}

Bates, D. R. and Damgaard, A.: 1949, Phil. Trans. Roy. Soc. A242, 101.

Belling, J.: 1970, Thesis, University of London.

Burgess, A., Hummer, D. G., and Tully, J. A.: 1970, Phil. Trans. Roy. Soc. A266, 225.

Burgess, A. and Percival, I. C.: 1968, Adv. Atomic Molec. Phys. 4, 109.

Eissner, W. and Nussbaumer, H. : 1969, J. Phys. B. [2], 2, 1028.

Godfredsen, E.: 1966, Astrophys. J. 145, 308.

Layzer, D.: 1959, Ann. Phys. (U.S.A.) 8, 271.

Layzer, D. and Garstang, R. H.: 1968, Ann. Rev. Astron. Astrophys. 6, 449.

Saraph, H. E., Seaton, M. J., and Shemming, J.: 1969, Phil. Trans. Roy. Soc. A264, 77.

Stewart. J. C. and Rotenberg, M.: 1965, Phys. Rev. 140, A1 508.

Van Regemorter, H.: 1962, Astrophys. J. 136, 906.

Wiese, W. L., Smith, M. W., and Glennon, B. M.: 1966, Atomic Transition Probabilities, NSRDSNBS4 - U.S. Govt. Printing Office, Washington.

\section{DISCUSSION}

L. H. Aller: Is it possible to calculate collision strengths for equivalent $d$-electrons, relevant for ions in various stages of ionization, e.g. (FevII)? If there are difficulties, do these arise from computational or from conceptual difficulties? That is, do we need some new physics?

$M$. J. Seaton: No new physics is needed. No calculations have yet been made for systems with equivalent $d$-electrons, but I think that we are now reaching the stage at which such calculations could be attempted.

Note added in proof. Calculations for Fevil have now been made by W. Eissner and $\mathrm{H}$. Nussbaumer. 